\title{
Simulation and design of arrayed waveguide gratings for InP membranes using an efficient numerical method and improved shallow to deep transitions
}

\author{
Citation for published version (APA): \\ Gargallo, B., Jiao, Y., Muñoz, P., van der Tol, J. J. G. M., \& Leijtens, X. J. M. (2016). Simulation and design of \\ arrayed waveguide gratings for InP membranes using an efficient numerical method and improved shallow to \\ deep transitions. Optical and Quantum Electronics, 48, 1-11. [356]. https://doi.org/10.1007/s11082-016-0628-5
}

DOI:

10.1007/s11082-016-0628-5

Document status and date:

Published: 17/06/2016

Document Version:

Publisher's PDF, also known as Version of Record (includes final page, issue and volume numbers)

\section{Please check the document version of this publication:}

- A submitted manuscript is the version of the article upon submission and before peer-review. There can be important differences between the submitted version and the official published version of record. People interested in the research are advised to contact the author for the final version of the publication, or visit the DOI to the publisher's website.

- The final author version and the galley proof are versions of the publication after peer review.

- The final published version features the final layout of the paper including the volume, issue and page numbers.

Link to publication

\footnotetext{
General rights

- You may freely distribute the URL identifying the publication in the public portal. follow below link for the End User Agreement:

www.tue.nl/taverne

\section{Take down policy}

If you believe that this document breaches copyright please contact us at:

openaccess@tue.nl

providing details and we will investigate your claim.
}

Copyright and moral rights for the publications made accessible in the public portal are retained by the authors and/or other copyright owners and it is a condition of accessing publications that users recognise and abide by the legal requirements associated with these rights.

- Users may download and print one copy of any publication from the public portal for the purpose of private study or research.

- You may not further distribute the material or use it for any profit-making activity or commercial gain

If the publication is distributed under the terms of Article $25 \mathrm{fa}$ of the Dutch Copyright Act, indicated by the "Taverne" license above, please 


\title{
Simulation and design of arrayed waveguide gratings for InP membranes using an efficient numerical method and improved shallow to deep transitions
}

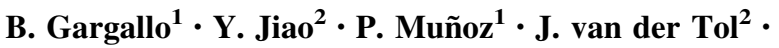 \\ X. Leijtens ${ }^{2}$
}

Received: 7 August 2015/ Accepted: 10 June 2016

(C) Springer Science+Business Media New York 2016

\begin{abstract}
We analyze the arrayed waveguide grating response on indium phosphide (InP) membranes on silicon (IMOS) technology. The model is based on an analytical approach (Kleijn et al. in Nat Photon 1:303-305, 2013) that provides a better accuracy than the Gaussian approximation for similar simulation times. Improved shallow to deep transitions to avoid possible layer misalignments are also presented and simulated.
\end{abstract}

Keywords Arrayed waveguide grating · Integrated optics devices · Wave optics

\section{Introduction}

The arrayed waveguide grating (AWG) is one of the most important devices in optical communication networks. It has been used extensively in wavelength-division multiplexing (WDM) systems, due to its multiplexing and demultiplexing functionalities (Smit and van Dam 1996). In these networks, components are required to provide stable operation while a reproducible fabrication process is mandatory. For these reasons, photonic integration is envisaged as the solution since it provides the necessary requirements.

This article is part of the Topical Collection on Optical Wave \& Waveguide Theory and Numerical Modelling, OWTNM' 15.

Guest edited by Arti Agrawal, B.M.A. Rahman, Tong Sun, Gregory Wurtz, Anibal Fernandez and James R. Taylor.

\section{B. Gargallo}

bergarja@iteam.upv.es

1 iTEAM Research Institute, Universitat Politècnica de València, Valencia, Spain

2 COBRA Research Institute, Eindhoven University of Technology, Eindhoven, The Netherlands 
Traditionally fabricated on silica on silicon integration technology (Dragone et al. 1991), it is possible to find examples of AWGs in almost any material platform (indium phosphide, silicon on insulator, silicon nitride, aluminium gallium arsenide...). The cost in photonic integrated circuits (PICs) is directly related to its footprint (Kirchain and Kimerling 2007), so technologies with higher index contrast where the field is highly confined in the waveguide and bending radius can be decreased are preferred to obtain smaller devices with the same functionality. As a drawback, devices are more sensitive to fabrication errors, as for example waveguide sidewall roughness or layer misalignments.

The IMOS photonic integration technique, based on the use of an indium phosphide (InP) membrane on top of a silicon chip provides all the advantages derived from high index contrast (HIC) technologies, but also the possibility of integrating active and passive devices with approximately the same dimensions as silicon on Insulator (SOI) technologies (van der Tol et al. 2014).

The main problem when modelling AWGs is the difficulty to find closed analytical equations describing its operation, being possible to find in the literature several models using Fourier optics (Dragone 1989; Muñoz et al. 2002). However, the Gaussian field approximation of the fundamental mode fails to predict the exponential tails of the field. It is also possible to keep the behaviour of the field using exponential and cosine functions, applying the EIM as in Kleijn et al. (2013). Nonetheless, the use of the effective index method (EIM) is not typically accurate in HIC technologies.

In this paper, we report how to apply successfully the analytical model in Kleijn et al. (2013) in HIC technologies as IMOS, obtaining a very fast and accurate simulation tool for double-etched AWGs. We also provide the design and simulations for a shallow to deep transition applicable in HIC technologies with reduced losses and high fabrication tolerance.

\section{Theoretical model}

The AWG is a passive device composed of groups of waveguides and free propagation regions. In the most common shape (Fig. 1), two free propagation regions (FPRs) are connected by a group of waveguides called arrayed waveguides. Between these arrayed

Fig. 1 Arrayed waveguide grating schematic. FPR free propagation region

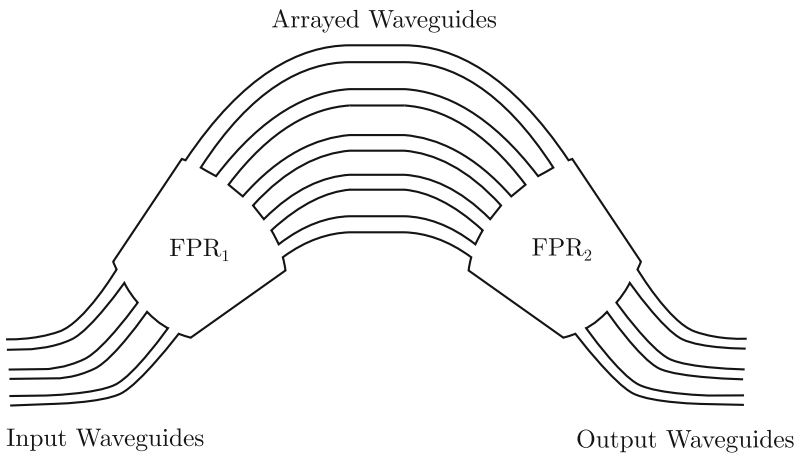


waveguides exist an incremental length that introduces a linear phase front in the input signal. The light with the introduced phase shift in combination with the second free propagation region will focus the different spatial positions.

To analyze the AWG it is necessary to know the field shape at the waveguides, since the length of the free propagation regions and the final passband shape will be determined by it. The effective index method (EIM) (Knox and Toulios 1970), an extension of Marcatili's method (Marcatili 1969), is probably the most simple method to analyze optical waveguides. The main idea underlying this method is to replace the rectangular waveguide structure by a slab waveguide (with infinite width in one direction) and then use the calculated effective index to replace the waveguide to a slab in the orthogonal direction. In the typical waveguides, it can be explained as follows: first, the optical waveguide is divided in three regions (slab, core, slab) calculating the effective index for each part. Then, a three layer slab in the orthogonal direction using the previously calculated effective indexes is solved, obtaining the approximation for the waveguide effective index.

The main limitation when using this method is the restriction to solve only low index contrast structures, where the difference in refractive index between the used materials is low and the aspect ratio is high enough to have almost all the field transverse variation in the wider dimension. Thus, for one chosen technology, the accuracy of the method will improve increasing the aspect ratio (i.e. having more slablike waveguides) or increasing the normalized frequency $V$, defined as $V=$ $b k n_{1}\left(\Delta_{2} / 2\right)^{1 / 2}$ (Chiang 1991). In the previous equation $b$ is the waveguide thickness, $k$ is the wavenumber defined as $k=2 \pi / \lambda$ and $\lambda$ is the wavelength, $n_{1}$ is the core refractive index and $\Delta_{2}$ is the relative index step defined as $\Delta_{2}=\left(n_{1}^{2}-n_{2}^{2}\right) /\left(2 n_{1}^{2}\right)$ with $n_{2}$ the substrate refractive index. For this reason, as typically the cross-section and wavelength are fixed, the EIM could be applicable if the aspect ratio is increased (i.e. using wider waveguides).

High index contrast structures have a reduced cross-section, where the thickness of the waveguide is comparable to the width, so the EIM is not the best choice in terms of accuracy. On the other hand, particular HIC structures can also be analyzed using this method when special layerstacks are used, as in the case of the typical COBRA deepetched cross-section where the cladding and substrate have a refractive index very similar to the core (Smit et al. 2014).

To obtain the mode profile approximation, it is necessary to reduce the $3 \mathrm{D}$ waveguide to a 2D waveguide applying the EIM and use a mode solver to obtain the guided modes. In the case of this paper, the scalar approximation of the EIM is used, in order to obtain reduced calculation times. Thus, the field profile can be approximated as cosine and exponential functions (Kleijn et al. 2013):

$$
U(x)=C \begin{cases}\cos \left(\frac{u}{2}-\phi\right) \exp \left(\frac{v}{2}+\frac{v x}{d}\right) & \text { for } x<-\frac{d}{2} \\ \cos \left(\frac{u x}{d}+\phi\right) & \text { for }|x| \leq \frac{d}{2} \\ \cos \left(\frac{u}{2}+\phi\right) \exp \left(\frac{w}{2}-\frac{w x}{d}\right) & \text { for } x>\frac{d}{2}\end{cases}
$$

with 


$$
\begin{aligned}
& v=k_{0} d\left(N^{2}-n_{2}^{2}\right)^{1 / 2} \\
& u=k_{0} d\left(n_{1}^{2}-N^{2}\right)^{1 / 2} \\
& w=k_{0} d\left(N^{2}-n_{0}^{2}\right)^{1 / 2} \\
& \phi=\frac{1}{2} \begin{cases}\arctan \left(\frac{w}{u}\right)-\arctan \left(\frac{v}{u}\right) & \text { for } \mathrm{TE} \\
\arctan \left(\frac{n_{1}^{2} w}{n_{0}^{2} u}\right)-\arctan \left(\frac{n_{1}^{2} v}{n_{2}^{2} u}\right) & \text { for } \mathrm{TM}\end{cases}
\end{aligned}
$$

where $k_{0}=2 \pi / \lambda_{0}, m$ is the mode number, $N$ is the mode index, $x$ is the spatial position and $d$ is the waveguide width. The mode is normalized through the coefficient $C$, obtained from the power normalization:

$$
1=\int_{-\infty}^{\infty} U(x) U^{*}(x) d x
$$

and can be expressed as:

$$
C=\sqrt{\frac{2}{d}}\left[1+\frac{\cos \left(\frac{u}{2}-\phi\right)^{2}}{\operatorname{Re}(v)}+\frac{\sin (u) \cos (2 \phi)}{u}+\frac{\cos \left(\frac{u}{2}+\phi\right)^{2}}{\operatorname{Re}(w)}\right]^{-1 / 2}
$$

This equations are valid only for small index differences between the ribs, so it is only reliable for the shallow waveguides when HIC cross-sections are used. Typical AWGs are designed to be double-etched, what means that the free propagation regions (FPRs) will be fabricated in the shallow cross-sections and also their input/ outputs, while the arrayed waveguides (where the incremental length will be introduced) should be fabricated in deeply etched cross-sections to reduce the losses. The main reason for such structure is to reduce the losses and the possible reflections in the interface between the FPR and the waveguides, since deeply etched waveguides cause a large discontinuity in this interface (van Dam et al. 1996; den Besten et al. 2002).

However, the case of a single-etched AWG using a HIC technology is not evaluated yet. As was commented before, the EIM is not suitable for this type of waveguides. Nonetheless, it is possible to find in the literature several approximations to predict the field in the waveguides, being the most appropriate the one in Westerveld et al. (2012). The main idea is to use the full Marcatili's approximation making the ansatz that the field can be spatially separated in two axis and it can be approximated as a product of sine, cosine and exponential functions. This idea, from which the EIM is an approximation, enables to obtain an accurate field profile approximation for deep-etched waveguides for HIC technologies, as is the case of SOI or IMOS. Moreover, the sine and exponential approximation is fully compatible with the model presented in Kleijn et al. (2013), obtaining a very accurate and efficient model for AWGs fabricated in SOI or other HIC technologies. 


\section{Design and simulation}

\subsection{Field profile approximation}

In IMOS technology, the cross-section consists of a silicon substrate, a $1850 \mathrm{~nm}$ height layer of silicon dioxide and the InP membrane (300 nm height) where the waveguides will be fabricated. A schematic of the two different cross-sections (deep and shallow) is shown in Fig. 2. As a waveguide parameters, shallowly etched waveguides with a $2.0 \mu \mathrm{m}$ width are used. Then, Eq. 1 is used to calculate the TE (electric field parallel to the $\mathrm{x}$ axis) field profile.

As a comparison, Fig. 3 shows the real field profile obtained from a commercial software (http://www.phoenixbv.com) using the Film Mode Matching (FMM) method and the different approximations using a Gaussian profile and the EIM. From this comparison, it is possible to see that the EIM approximates very well the real profile inside the core, while the exponential tails are underestimated. It is also clear that the Gaussian approximation introduces an error when fitting the field profile since it underestimates the mode width inside the core and overestimates the exponential tails. However, the error introduced by the EIM is low when the waveguide aspect ratio is high, as in the case of the shallow crosssection.

The EIM can also be applied in the case of deep waveguides, at expenses of a higher approximation error. Figure 4 shows the case when deep waveguides with a width of $2.0 \mu \mathrm{m}$, Fig. $4 \mathrm{a}$, and a width of $0.4 \mu \mathrm{m}$, Fig. $4 \mathrm{~b}$, are simulated. The comparison shows how when wide deep waveguides are used, the EIM approximates correctly the field in the core of the waveguide, but it fails to predict the exponential tails. Moreover, the Gaussian approximation fails to approximate the field in every part. In the second case, when simulating narrow waveguides, both approximations have a large error when approximating the strong kinks obtained due to the high index contrast in the interface between the InP material and air.

Then, in the case of using HIC waveguides with a low aspect ratio (i.e. narrow widths), the sine and exponential approximation presented in Kleijn et al. (2013) fails. As was commented before, other models based on Marcatili's method could be used, as in the case of Westerveld et al. (2012), obtaining a cosine, sine and exponential approximation that is fully compatible with the AWG model in Kleijn et al. (2013).

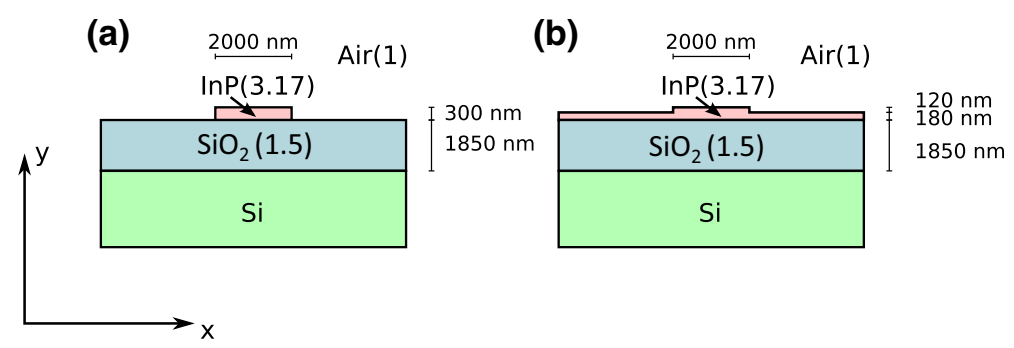

Fig. 2 IMOS cross-sections for a deep waveguide and $\mathbf{b}$ shallow waveguide. The refractive index for each material is indicated in parenthesis for the central wavelength $\lambda_{0}=1.55 \mu \mathrm{m}$ 


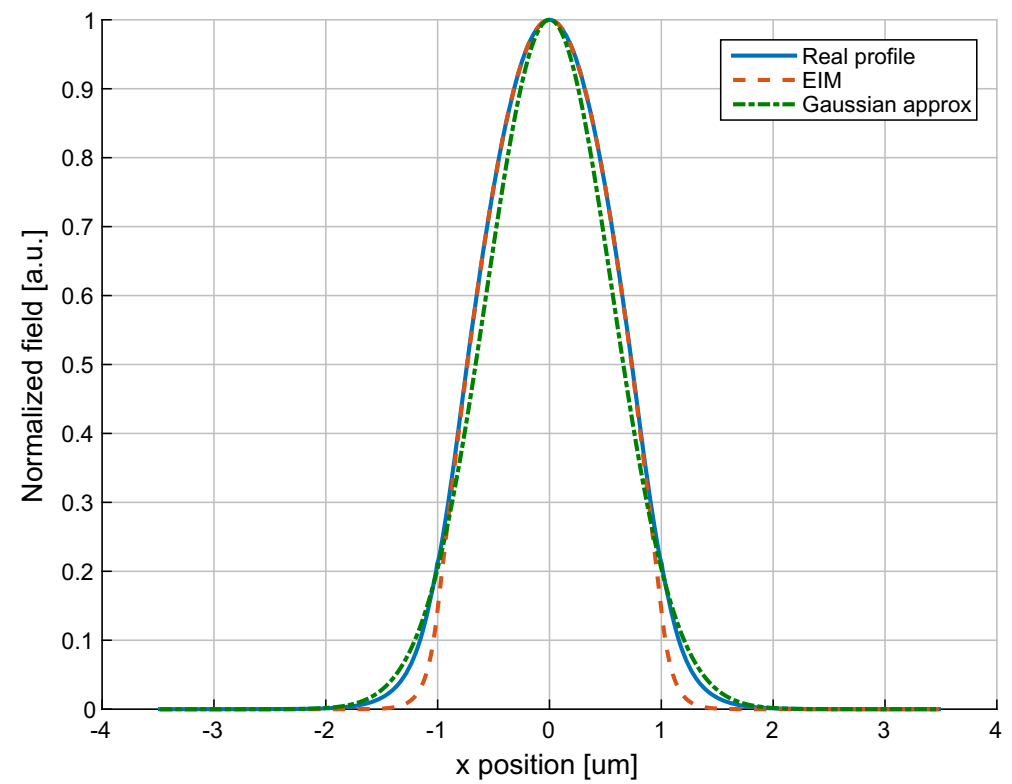

Fig. 3 Comparison between the real profile and EIM and Gaussian approximations for the shallow waveguide

(a)

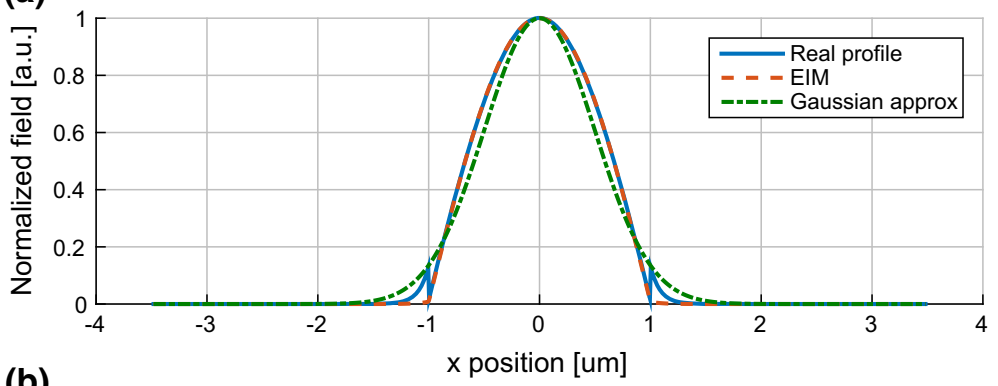

(b)

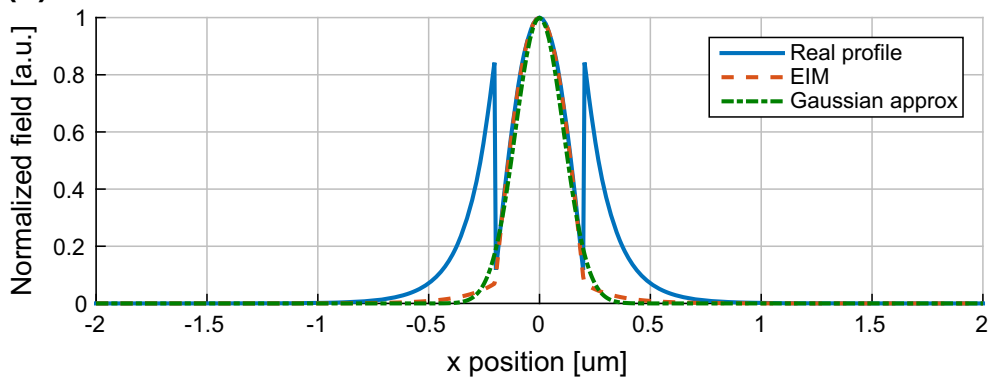

Fig. 4 Comparison between the real profile and EIM and Gaussian approximations for a $2.0 \mu \mathrm{m}$ width and b $0.4 \mu \mathrm{m}$ width deep waveguides 


\subsection{AWG simulation}

To check the validity of the model, an AWG for IMOS technology was designed and simulated. The AWG parameters are the following: the center wavelength is $1550 \mathrm{~nm}$, using four channels with a spacing of $3.2 \mathrm{~nm}$ and a FSR of $12.8 \mathrm{~nm}$. Regarding the waveguides, $2.0 \mu \mathrm{m}$ width shallow waveguides are used as input/outputs of the FPRs, while the arrayed waveguides are designed using $0.8 \mu \mathrm{m}$ width deep waveguides with an effective index of 2.5492. The calculated focal length is $48.71 \mu \mathrm{m}$, the incremental length between AWs is $50.47 \mu \mathrm{m}$, the number of AWs is 13 and the bend radius was set to $5 \mu \mathrm{m}$. The transfer function was computed for the real profile using the Fresnel diffraction integral and the EIM approximation with the model in Kleijn et al. (2013). This model assumes that the input and output waveguides in each free propagation region are positioned following the Rowland circle mounting and the when the light exits the input waveguides it is diffracted. Then the field at the other side can be calculated through the $2 \mathrm{D}$ Fresnel-Huygens diffraction integral. Moreover, the coupling between the modes in the waveguides is given by the overlap integral between the output mode and the diffracted field (Kleijn et al. 2013).

The corresponding end-to-end transfer function for this AWG is depicted in Fig. 5. Note the simulations show losses of approximately $3.5 \mathrm{~dB}$ for the EIM approximation and lower than $3 \mathrm{~dB}$ for the real profile. We did not include the propagation loss in the waveguides for IMOS (typically around $4 \mathrm{~dB} / \mathrm{cm}$ ) and other detrimental effects as fabrication imperfections. From the simulations the $1-\mathrm{dB}, 3-\mathrm{dB}$ and $20-\mathrm{dB}$ bandwidths are $0.86,1.45$ and $3.51 \mathrm{~nm}$ respectively for the real profile simulation and $0.77,1.38$ and $3.35 \mathrm{~nm}$ for the EIM simulation. This difference in bandwidth can be explained as the EIM underestimates the field in the exponential tails. It is also clear from simulations that the noise level is around $5 \mathrm{~dB}$ higher in the case of the analytical model. In this case, this disparity comes from the

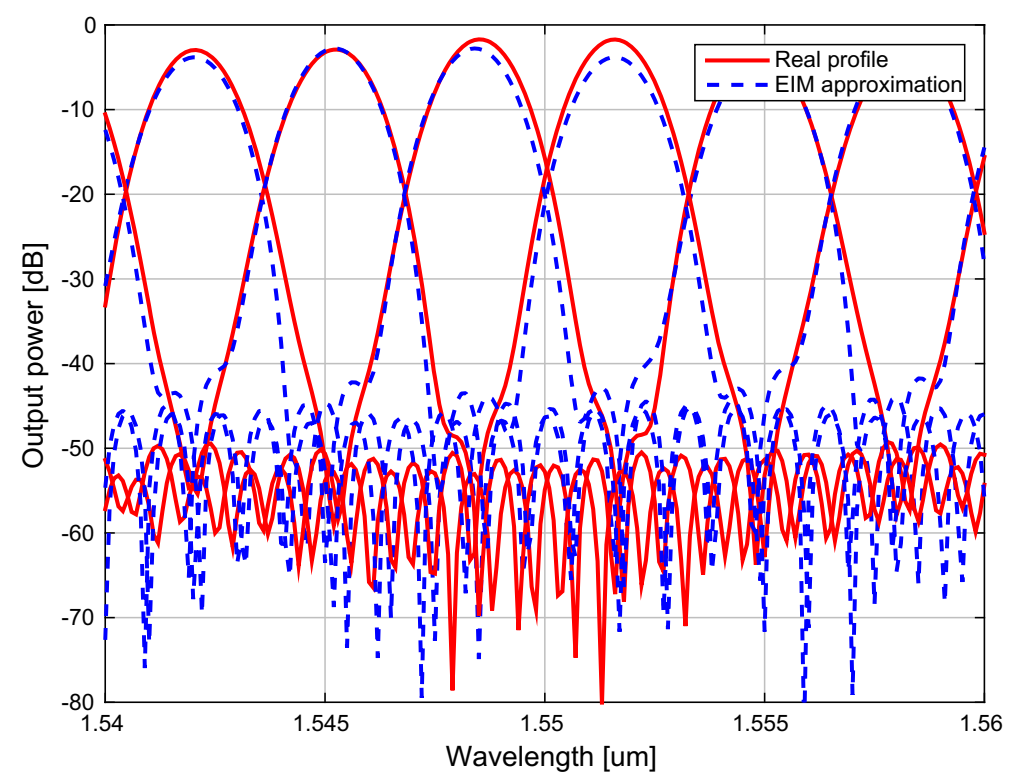

Fig. 5 Comparison between simulations 
analytical model where the overlap between modes of adjacent waveguides is taken into account.

In conclusion, the comparison between simulations using the real profile together with the Fresnel diffraction integral and the analytical approach shows a very good match, obtaining for 2000 points calculation times of $170.3 \mathrm{~s}$ in the case of the real profile and $1.5 \mathrm{~s}$ for the analytical model (simulations calculated using a intel core i5 processor).

\section{Shallow to deep transitions}

As was commented in the introduction, HIC technologies are very sensitive to fabrication errors, due to the strong confinement of the field in the waveguide. The most important contributions to these errors are the sidewall roughness in the waveguides and the misalignment between layers when using different cross-sections. In the case of AWGs, the sidewall roughness is a technology intrinsic factor, so it will depend on the fabrication process. However, when using a double-etched layout the layer misalignment rises as an important phase error contribution that can be avoided or minimized designing carefully the transitions between different cross-sections. In any case, the use of a shallow to deep transition at the input/output of the FPRs has two benefits: it reduces the losses and reflections in the interface between the FPR and the waveguides as was discussed before, and it permits to use the field approximation described in Sect. 2 if the transition is correctly designed to avoid high order modes at the end of the shallow waveguide.

In the case of this paper, the shallow to deep transitions are based on the butt-coupled transitions in Maat (2001). This transition has the advantage to be very tolerant to the misalignments when fabricating. Figure 6 shows the schematic of such transition. It consists of a shallow cross-section that is widened to an interface width. This interface will be butt-coupled to a deep waveguide, where typically the field is slightly more confined in the core, and linearly tapered to the desired final deep width. When using this structure as shallow to deep transition, any misalignment will cause only a displacement of the shallow slopes, which it will not affect to the performance if the interface length is designed to be

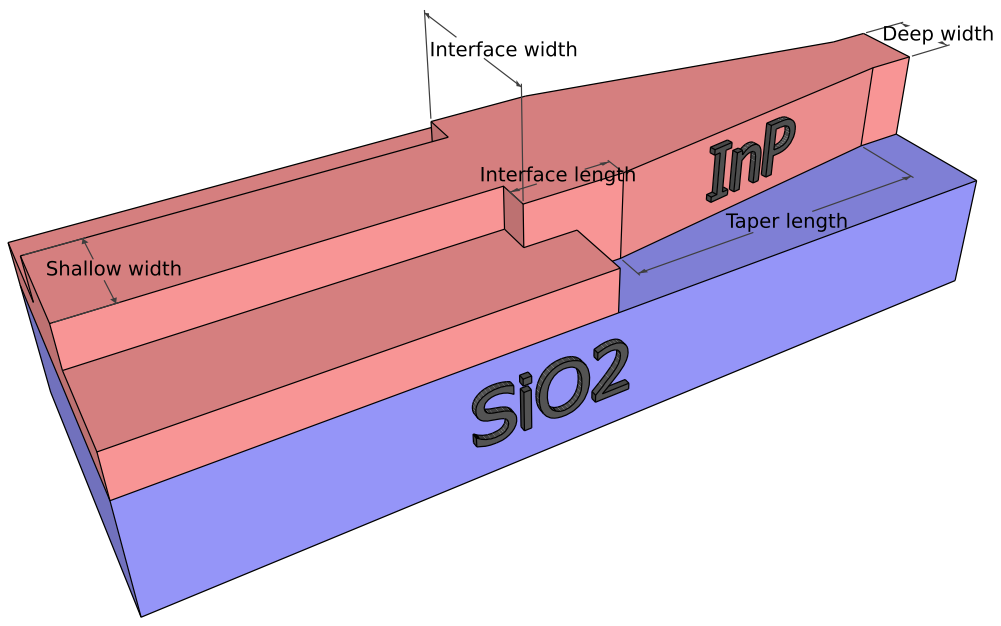

Fig. 6 Shallow to deep transition schematic 
longer than the foundry alignment error. The most critical part of this transitions is the design of the interface between the shallow and deep waveguides, since the mode profile is different for each cross-section. From theory, the slope in shallow waveguides causes that the field width is higher than in a deep waveguides for the same waveguide width. For this reason, different simulations calculating the overlap between the modes are performed to choose the best case. Although the fields will be more similar when wide waveguides are used, it is necessary a compromise between size and losses due to coupling.

Simulations are performed using a 3D propagation tool using the EigenMode Expansion (EME) method (http://www.photond.com). The simulation results in Fig. 7 show that the best case in terms of propagation and reflection when simulating the interface between both cross-sections is obtained using a slightly wider transition (deep) waveguide in comparison with the shallow waveguide. The interface length is fixed to $3 \mu \mathrm{m}$ to avoid any layer misalignment.

As a fixed parameters, the shallow and deep widths are fixed to 2 and $0.8 \mu \mathrm{m}$, respectively. Simulations of the total structure including the linear taper and different interface widths are shown in Fig. 8. From there, the best case is chosen to be using an interface width of $2.1 \mu \mathrm{m}$ and a taper length of $24 \mu \mathrm{m}$, obtaining a losses of only $0.02 \mathrm{~dB}$ per transition. In any case, it is possible to use the same width for the shallow and the transition sections since simulations show additional losses of $0.02 \mathrm{~dB}$, being not very critical.
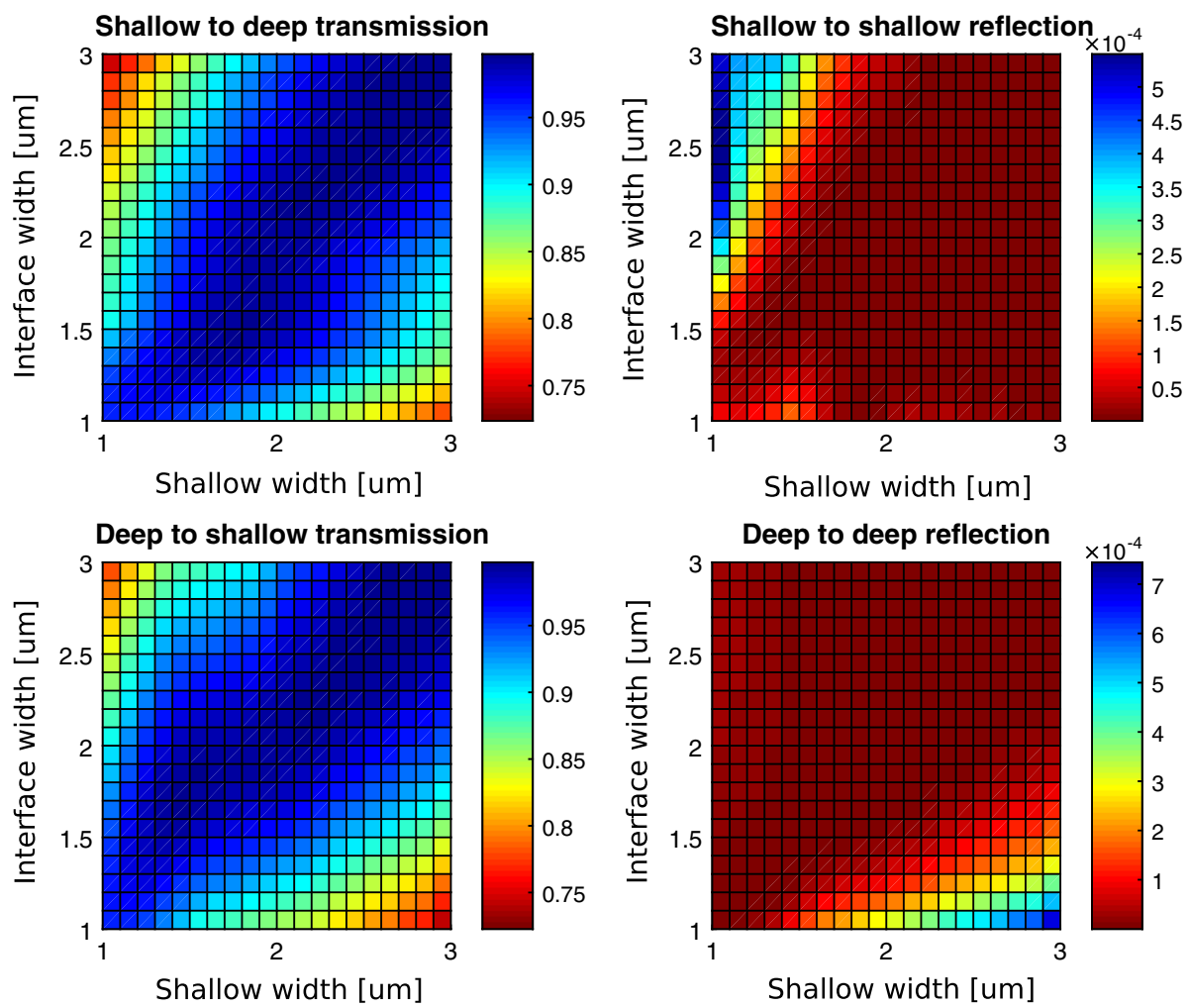

Fig. 7 Shallow to deep transition simulations using the fundamental TE mode 

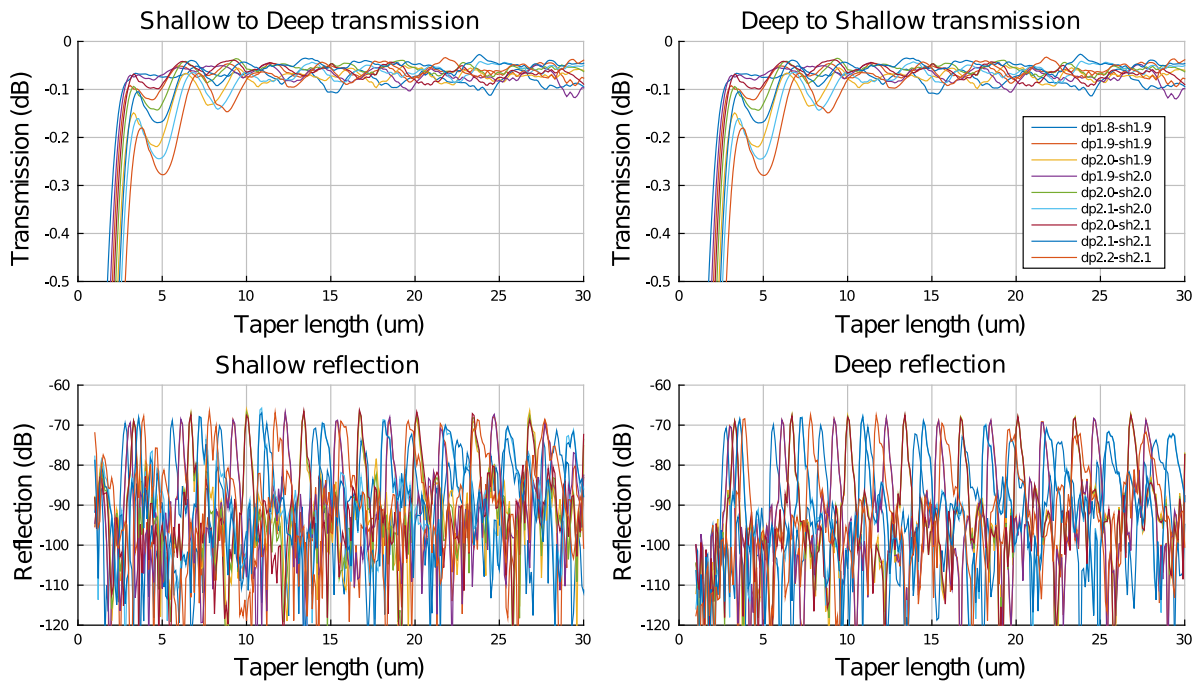

Fig. 8 Shallow to deep transition simulations using the fundamental TE mode

Although the shallow to deep transitions simulated are not very long, it is possible to reduce the size of such transition replacing the linear taper with a parabolic taper (Milton and Burns 1977).

\section{Conclusion}

In conclusion, the analytical model provided in Kleijn et al. (2013) is suitable for IMOS AWGs when using shallow waveguides as input/outputs, obtaining a good approach in a few seconds. The model is not only restricted to this technology, being also feasible in other HIC technologies as SOI. It is also possible to apply the field profile approximation in Westerveld et al. (2012) and follow the previously cited analytical model if only deeply etched waveguides are used. Shallow to deep transitions using a butt-coupled layout tolerant to layer misalignments are simulated, obtaining a robust structure in terms of layer misalignments and low losses.

Acknowledgments B. Gargallo acknowledges financial support through FPI Grant BES-2011-046100.

\section{References}

Chiang, K.: Performance of the effective-index method for the analysis of dielectric waveguides. Opt. Lett. 16, 714-716 (1991)

den Besten, J., Dessens, M., Herben, C., Leijtens, X., Groen, F., Leys, M., Smit, M.: Low-loss, compact, and polarization independent PHASAR demultiplexer fabricated by using a double-etch process. Photon. Technol. Lett. 14, 62-64 (2002)

Dragone, C.: Efficient NxN star couplers using Fourier optics. J. Lightw. Technol. 7, 479-489 (1989)

Dragone, C., Edwards, C.A., Kistler, R.C.: Integrated optics NxN multiplexer on silicon. IEEE Photon. Technol. Lett. 3, 896-899 (1991)

FIMMPROP, Photon Design, http://www.photond.com 
Kirchain, R., Kimerling, L.: A roadmap for nanophotonics. Nat. Photon. 1, 303-305 (2007)

Kleijn, E., Smit, M., Leijtens, X.: New analytical arrayed waveguide grating model. J. Lightw. Technol. 31, 3309-3314 (2013)

Knox, R., Toulios, P.: Integrated circuits for the millimeter through optical frequency range. In: Proceedings of MRI Symposium of Submillimeter Waves, ed. Polythecnic, New York (1970)

Maat, P.: InP-based integrated MZI switches for optical communication, Ph.D. Thesis (2001)

Marcatili, E.: Dielectric rectangular waveguide and directional coupler for integrated optics. Bell Syst. Tech. J. 48, 2071-2102 (1969)

Milton, A., Burns, W.: Mode coupling in optical parabolic horns. IEEE J. Quant. Electron. 13, 828-835 (1977)

Muñoz, P., Pastor, D., Capmany, J.: Modeling and design of arrayed waveguide gratings. J. Lightw. Technol. 20, 661-674 (2002)

OptoDesigner $^{T M}$, PhoeniX Software BV, http://www.phoenixbv.com

Smit, M., et al.: An introduction to InP-based generic integration technology. Semicond. Sci. Technol. 29, 083001 (2014)

Smit, M., van Dam, C.: Phasar-based WDM-devices: principles, design and applications. J. Sel. Topics Quantum Electron. 2, 236-250 (1996)

van Dam, C., Staring, A., Jansen, E., Binsma, J., van Dongen, T., Smit, M., Verbeek, B.: Loss reduction for phased-array demultiplexers using a double etch technique. In: Integrated Photonics Research 6 paper IMC6, OSA Technical Digest Series (1996)

van der Tol, J., Pello, J., Bhat, S., Jiao, Y., Heiss, D., Roelkens, G., Ambrosius, H., Smit, M.: Photonic integration in indium-phosphide membranes on silicon (IMOS). In: Proc. SPIE 8988, Paper 89880M (2014)

Westerveld, W., Leinders, S., van Dongen, K., Urbach, H., Yousefi, M.: Extension of Marcatili's analytical approach for rectangular silicon optical waveguides. J. Lightw. Technol. 30, 2388-2401 (2012) 